\title{
Determination of composition and structure of spongy bone tissue in human head of femur by Raman spectral mapping
}

\author{
M. Kozielski • T. Buchwald • M. Szybowicz • \\ Z. Błaszczak $\cdot$ A. Piotrowski $\cdot$ B. Ciesielczyk
}

Received: 22 April 2011/Accepted: 16 May 2011/Published online: 31 May 2011

(C) The Author(s) 2011. This article is published with open access at Springerlink.com

\begin{abstract}
Biomechanical properties of bone depend on the composition and organization of collagen fibers. In this study, Raman microspectroscopy was employed to determine the content of mineral and organic constituents and orientation of collagen fibers in spongy bone in the human head of femur at the microstructural level. Changes in composition and structure of trabecula were illustrated using Raman spectral mapping. The polarized Raman spectra permit separate analysis of local variations in orientation and composition. The ratios of $v_{2} \mathrm{PO}_{4}{ }^{3-} / \mathrm{Amide}$ III, $v_{4} \mathrm{PO}_{4}{ }^{3-} /$ Amide III and $v_{1} \mathrm{CO}_{3}{ }^{2-} / v_{2} \mathrm{PO}_{4}{ }^{3-}$ are used to describe relative amounts of spongy bone components. The $v_{1} \mathrm{PO}_{4}{ }^{3-}$ /Amide I ratio is quite susceptible to orientation effect and brings information on collagen fibers orientation. The results presented illustrate the versatility of the Raman
\end{abstract}

The samples were prepared at the Medical University in Poznan. All the measurements were carried out on Renishaw inVia microscope at the Poznan University of Technology. All experimental procedures were approved by the local bioethical commission at the Wielkopolska Medical Chamber in Poznan.

M. Kozielski · T. Buchwald $(\bowtie) \cdot$ M. Szybowicz Faculty of Technical Physics, Poznan University of Technology, Nieszawska 13a, 60-965 Poznań, Poland

e-mail: scabby@poczta.onet.pl

Z. Błaszczak

Faculty of Physics, Adam Mickiewicz University,

Umultowska 85, 61-614 Poznań, Poland

A. Piotrowski

Department of Anatomy, Poznan University of Medical

Sciences, Święcickiego 6, 60-784 Poznań, Poland

B. Ciesielczyk

Department of Surgery, Franciszek Raszeja Memorial Hospital,

Mickiewicz 2, 60-834 Poznań, Poland method in the study of bone tissue. The study permits better understanding of bone physiology and evaluation of the biomechanical properties of bone.

\section{Introduction}

Bone tissue is the main component of the skeletal system. There are different types of tissues and other structures that can be distinguished within the bone [1]. Important constituents of the bone are adipose tissue and the other hematopoietic tissues, along with other structures such as cartilage, articulate surfaces of the joints, periosteum, nerves and vessels. Macroscopically, the bone tissue can be divided into compact bone and spongy bone [1-3]. The compact bone is more resistant to mechanical forces than spongy bone and it is composed of osteons (osseous laminae arranged in a concentric pattern around Haversian canals). The spongy bone is composed of a network of trabecula of different shape and thickness arranged along the directions of action of mechanical pressure forces [2]. Therefore, the main protective function of spongy bone is its elasticity in preventing workload. Epiphysis and metaphysis of long bones are mainly composed of spongy bone. The bone is a unique type of connective tissue composed of certain types of cells differentiated from mesenchymal line such as pluripotential osteogenic cells, osteoblasts, osteocytes; and cells differentiated from hematopoietic line such as pro-osteoclasts and osteoclasts and an extracellular matrix which consists of an organic matrix and mineral components (apatite crystals, calcium salts and water) [4-6]. The organic matrix is made in $90 \%$ by type I collagen fibers [7]. The collagen fibers are more elastic and resistant to fractures than the apatite crystals. Therefore, the bone structure strength is determined by 
the mineral components [8], whereas the elasticity and toughness of the tissue-by the organic components [9]. Complementary relations between the mineral and the organic components ensure the optimal mechanical properties of bone [10]. Changes in tissue organization and composition cause differences in strength and biomechanical properties of the bone. The amount and quality of mineral and organic components, orientation and crosslinking of the collagen fibers, contribute to the quality of both types of bone tissues [11]. The arrangement of collagen fibers and apatite crystals and their composition in the trabecula can be determined at the microstructural level. Each trabecula is characterized by different arrangement of collagen fibers, which is understood in this work as different orientation of collagen fibers. In spongy bone tissue the process of organization and composition is more intense than in compact bone tissue because spongy bone is metabolically more active [12]. Osteoporosis is the main reason for decrease in bone mass in senior population. The frequency of bone fractures is higher in elderly population, the fractures happen unexpectedly during common daily activities [13, 14]. A frequent site of typical osteoporotic fracture is below the head of the femur. The orthopaedic surgery is the procedure of choice in osteoporotic fractures. The fracture of proximal end of femur is a serious problem for geriatric medicine [15].

An array of analytical tools including infrared and Raman spectroscopy have been used to study biological material [16]. The use of vibrational spectroscopies in biological investigation offers several advantages. Firstly, these physical techniques give information about the structure and composition of material studied on the microstructural level. Secondly, spectroscopic data are obtained in a non-invasive manner, so the same sample can be examined by various analytical methods. Thirdly, there is no limit to the size, allowing a study of large area of a sample [17]. Although the two major techniques offer similar outcomes, Raman analysis has definite advantages. Raman spectroscopy offers superior spatial resolution $0.6-1 \mu \mathrm{m}$ compared to infrared spectroscopy 5-10 $\mu \mathrm{m}$. In this way Raman techniques enable analysis of biologically important sites such as individual cement lines, individual lamellae, regions around micro cracks, and human dentin tubules, which would be impossible by infrared techniques $[18,19]$. Infrared absorption of biological molecules, particularly water, makes investigation very difficult [20]. In contrast to infrared spectroscopy, Raman spectroscopy is relatively insensitive to water, allowing analysis of fully hydrated samples, with minimal sample preparation. Nowadays, the newest Raman instruments are able to investigate most of the biological samples, especially bone tissue, in the visible range without fluorescence effect [16].
Because of heterogeneous nature of bone, a single point in Raman microspectroscopy cannot sufficiently describe chemical composition and structure of the sample. For this reason, Raman spectral imaging is becoming increasingly popular for the study of complex organized systems, because it gives spatial information on samples [21, 22]. In earlier studies Raman microspectroscopy has been used to determine carbonate to phosphate ratios and phosphate to amide ratios in several tissue types [18, 19, 23]. Raman spectral mapping has been used to demonstrate the orientation of mineral and collagen components in osteonal lamellae of the cortical bone [24] and has been shown to permit imaging of two adjacent orthogonal planes in cortical in order to obtain 3D information [25]. Raghavan et al. have shown the conditions under which polarized Raman spectroscopy can be used to quantitatively measure mineral and matrix orientation in highly turbid bone tissue. Systematic errors in the orientation distribution calculations have been minimized by employing a high numerical aperture objective [26]. Cortical and spongy bone has not always been studied separately. Raman microspectroscopy has been applied to compare cortical and spongy bone tissue from the same femur and tibia of standard laboratory mice [27]. Other authors have presented the potential of Raman microspectroscopy for the intravital study of bone with using bone chamber [16]. The use of bone chamber in Raman studies allows monitoring changes in composition of bone and biomaterials in living animals over time.

\section{Materials and methods}

The study materials were five femur heads from patients who underwent osteoporotic fractures below the head of femur. The material from a group of five patients between 60 and 74 years of age was studied. The treatment of choice in the above mentioned patients was hemialloplastic procedure (bipolar prosthesis was applied). The crosssections of $5 \mathrm{~mm}$ in thickness were obtained from heads of femurs of these patients. In this work, the results of a study on one representative femur head from a 67 year old woman are presented. Since the head of femur consists mainly of spongy bone, this part of bone tissue attracted a great deal of attention. The study has got the approval of the local Bioethical Commission at the Wielkopolska Medical Chamber in Poznań (No 14/2008 from August 27, 2008).

In the present study, Raman microspectroscopy was employed to analyze the mineral and organic constituents and orientation of collagen fibers in spongy bone tissue of the human head of the femur. Changes in composition and structure of the spongy bone tissue were illustrated using maps of polarized Raman spectra. The results demonstrate 
the versatility of the Raman method as the analytical spectroscopic technique and provide insights into the organization of spongy bone tissue at the microstructural level. Determination of the composition and collagen fibers arrangement permits better understanding of bone physiology and evaluation of the biomechanical properties of bone. This investigation contributes to development of a method allowing identification of persons at risk of bone fractures.

All the measurements were carried out on Renishaw inVia microscope with diode pumped laser characterized by $500 \mathrm{~mW}$ power and emitting $785 \mathrm{~nm}$ infrared wavelength. Laser beam was focused on the sample through the long working distance of the $50 \times / 0.5$ objective. Multiple scattering in turbid bone tissue cause light depolarization and introduces errors in polarized Raman measurements. The use of the smallest depth of field allows minimization of depolarization effects, which is needed for determination of collagen orientation in bone tissue [26]. Therefore, the measurements were made under a microscope in the high confocality mode. Confocal system improved axial resolution and the depth of field with this objective was $2.2 \mu \mathrm{m}$. The air-cooled CCD Camera detector (Rencam) and $1200 \mathrm{~mm}^{-1}$ diffraction grating were used. At the beginning of each experimental session the laser was checked for alignment with the optical axis of the microscope and the spectral data (wavenumber and intensity) were verified with the use of $521 \mathrm{~cm}^{-1}$ band of a silicon internal sample. Thanks to the above procedure, the Raman spectra were obtained in the same spectral conditions. For bone studies, Raman spectra were recorded in the spectral ranges 200-1800 and $2800-3200 \mathrm{~cm}^{-1}$. The overall spectral resolution was better than $1 \mathrm{~cm}^{-1}$, while the microscope objective ensured a spatial resolution of $2 \mu \mathrm{m}$. During measurements, the sample received $50 \mathrm{~mW}$. The Raman maps of spongy bone tissue were obtained using the motorized in the three axes stage of the microscope allowing to monitor the sample through an optical camera. The Raman images were acquired in rectangular areas of $90 \times 90 \mu \mathrm{m}^{2}$ and at $10 \mu \mathrm{m}$ step size at five different sites of the spongy bone tissue of particular femur heads. The analyses of each map gave the same results. The incident light was linearly polarized optionally with a half-wave plate. The Raman scattered light could be detected in the linear polarization (vertical and horizontal direction) using polarisers. Polarization of scattered light allows determination of the orientation of collagen fibers. The Raman maps were obtained by single analysis of each data point. The time of exposure to get individual Raman spectra was $10 \mathrm{~s}$, the spectra were recoded without accumulation. Cosmic ray artefacts were removed and analyses of the spectra were performed in the same WIRE 3.0 (Renishaw) software. Rayleigh scattering background was subtracted manually from each raw spectrum by using the polynomial curve. The images which display changes in composition and orientation of collagen fibers in spongy bone tissue were generated by the OriginPro 8.0 software.

\section{Results and discussion}

Figure 1a shows a typical Raman spectrum of spongy bone tissue. The major bands in Raman spectra of bone tissue corresponding to mineral and organic constituents are labeled. Information about mineral and organic composition is obtained simultaneously, giving a complete arrangement of the bone constituents in the area surveyed. A very important fact is that the bands corresponding to these two phases are clearly separated [27]. Fig. 1b depicts the range of wavenumbers in which the bands in the Raman spectrum of bone can occur and specifies the bone structure components corresponding to these bands. The mineral part of
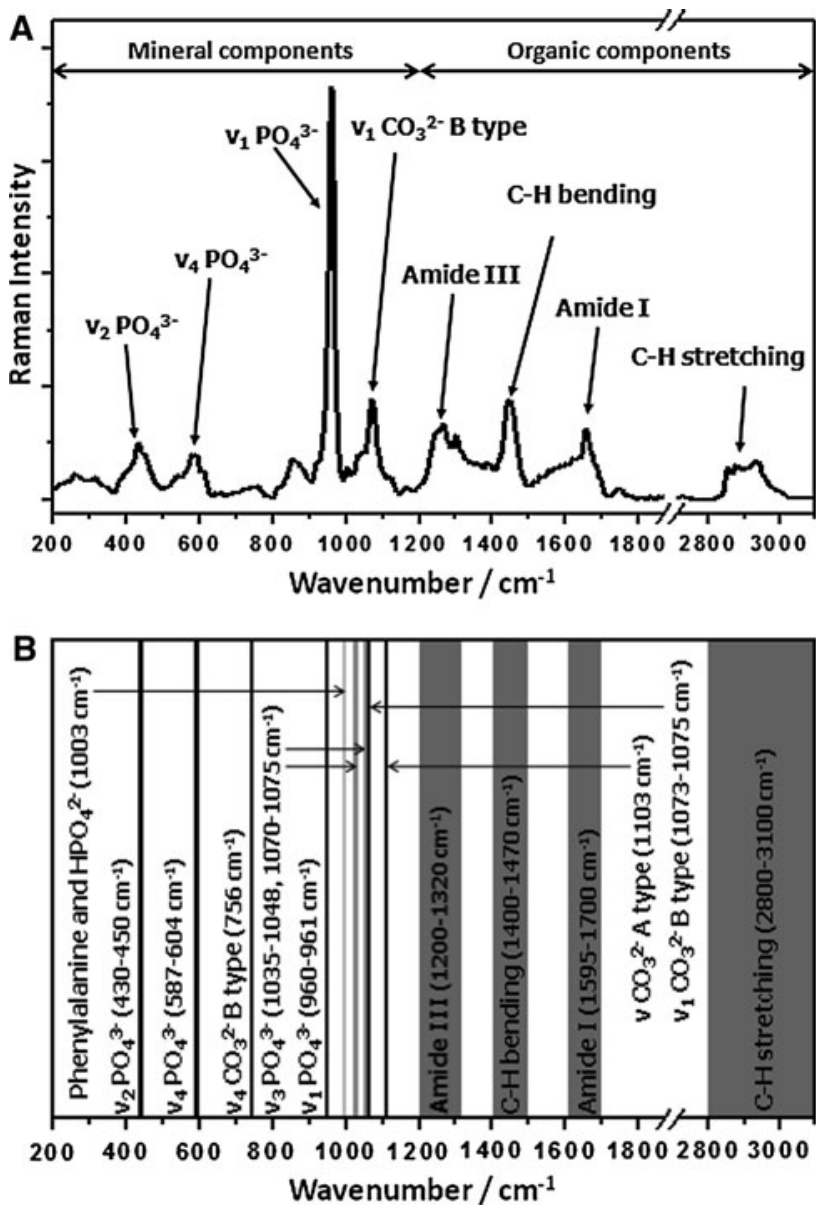

Fig. 1 Typical Raman spectrum of spongy bone tissue showing the major bands and the corresponding compounds. Background signal has been removed (a). The wavenumber ranges in which the bands can occur in the Raman spectrum of bone tissue (b) 
the spectrum is dominated by the $v_{1}$ phosphate $\left(\mathrm{PO}_{4}{ }^{3-}\right)$ internal mode at $961 \mathrm{~cm}^{-1}$. The other markers of the mineral component connected with the $v_{2}$ and $v_{4}$ phosphate vibrations are detected at 430 and $587 \mathrm{~cm}^{-1}$, respectively $[16,25$, 27]. The $v_{3}$ phosphate vibration is not well visible in the region 1035-1048 $\mathrm{cm}^{-1}$ and is overlapped with that of carbonate, which is substituted in the apatite structure, appearing in the region $1070-1075 \mathrm{~cm}^{-1}$, so it cannot be used to detect changes in the spongy bone tissue. Both B-type and A-type $\mathrm{CO}_{3}{ }^{2-}$ bands are observed at 1073 and $1103 \mathrm{~cm}^{-1}$, respectively [16]. However, the weakness of the A-type carbonate band does not permit getting information about the composition of spongy bone tissue, therefore B-type mode only is used. The major bands assigned to the organic components are found in region: $\sim 1200-1320 \mathrm{~cm}^{-1}$ (amide III), $\sim 1595-1700 \mathrm{~cm}^{-1}$ (amide I), $\sim 1400-1470 \mathrm{~cm}^{-1}$ and $\sim 2800-3100 \mathrm{~cm}^{-1}$ (bending and stretching modes of $\mathrm{C}-\mathrm{H}$ groups, respectively) [16,24]. In this study the maxima of the bands assigned to amide I and amide III are at 1655 and $1300 \mathrm{~cm}^{-1}$ respectively. The bands assigned to $\mathrm{C}-\mathrm{H}$ groups present in both collagenous and noncollagenous moieties, are visible at $1443 \mathrm{~cm}^{-1}$ and 2852, 2877, $2935 \mathrm{~cm}^{-1}$.

The Raman signal depends not only on the composition but also on the local orientation of collagen fibers or apatite crystals and changes according to the polarization of incident and scattered light, so interpretation of the spectra of bone tissues is more complicated than that of those of isotropic materials [24, 27, 28]. The collagen triple helix structure determines the positions of the amide bonds with respect to the backbone. The $\mathrm{C}=\mathrm{O}$ bonds are perpendicular to the molecular axis, while $\mathrm{C}-\mathrm{N}$ bonds occur in perpendicular and along this axis [24]. The Amide I band is predominantly associated with the $\mathrm{C}=\mathrm{O}$ stretching vibration, whereas the Amide III mode is mostly connected with C-N vibration [29]. Therefore, the behavior of polarized bands of Amide I and III is completely different. The intensity of Amide I band is higher for the polarization perpendicular to the collagen fibers, while the Amide III band is characterized by two different $\mathrm{C}-\mathrm{N}$ vibration modes corresponding to perpendicular and parallel conformations [30]. When both conformations are combined, no orientation effect is detectable [24]. The crystallographic $\mathrm{C}$-axis of apatite crystals is along the collagen fibers [11] and is associated with $v_{1}$ phosphate vibrations. Thus the $v_{1} \mathrm{PO}_{4}{ }^{3-}$ band intensity has a maximum value when the polarization of incident light is parallel to the orientation of collagen fibers [31]. Kazanci et al. [24] reported that Raman band intensities depend strongly on both chemical composition and collagen fibers orientation of cortical bone tissue with respect to the linear polarization of the incident light. This work has also shown that the bands, such as $v_{1} \mathrm{PO}_{4}{ }^{3-}$ and Amide I are quite sensitive to the orientation and the polarization of the incident light, so these bands give information about the orientation of collagen fibers. In contrast, the bands such as those assigned to Amide III, $v_{2} \mathrm{PO}_{4}{ }^{3-}$ and $v_{4} \mathrm{PO}_{4}{ }^{3-}$ are less sensitive to the orientation effects and indicate changes in mineral and organic composition of bone tissue. We are concerned with a similar but not the same problem. In our work Raman microspectroscopy was employed to determine chemical composition and orientation of collagen fibers in spongy bone tissue. In this study the linearly polarized incident and also scattered light was used to obtain Raman maps to show changes in the structure of trabecula surface.

The intensity of individual Raman bands cannot be used as an empirical measure of the content of mineral and organic components in bone tissue, because the irregularity of biological material surface strongly influences the bands intensity. During measurements the distance from the objective to the sample is changed, therefore the focus of the laser beam is also changed. In order to remove the impact of this factor, the ratios of intensities of the appropriate bands in the Raman spectra were employed. Figure 2 shows the line spectra of the (a) $v_{2} \mathrm{PO}_{4}{ }^{3-} /$ Amide III (b) $v_{4} \mathrm{PO}_{4}{ }^{3-} /$ Amide III, (c) $v_{1} \mathrm{PO}_{4}{ }^{3-} /$ Amide $\mathrm{I}$ and (d) $v_{1} \mathrm{CO}_{3}{ }^{2-} / v_{2} \mathrm{PO}_{4}{ }^{3-}$ ratios. This result is obtained from Raman maps of spongy bone tissue for different polarizations of the incident light. Fig. 2e illustrates the area of the bone tissue studied by Raman spectra with 10 measurement lines. The band intensities of the Raman spectra obtained from these lines are presented on ratio plots. The investigation was carried out over rectangular areas of $90 \times$ $90 \mu \mathrm{m}^{2}$ and the Raman spectra were acquired at $10 \mu \mathrm{m}$ step size.

As markers of chemical composition the ratios $v_{2} \mathrm{PO}_{4}{ }^{3-} /$ Amide III, $v_{4} \mathrm{PO}_{4}{ }^{3-} /$ Amide III and $v_{1} \mathrm{CO}_{3}{ }^{2-} / v_{2} \mathrm{PO}_{4}{ }^{3-}$ were used. The bands used in the above ratios are less sensitive to orientation effect as shown in Fig. 2a, b and d. The ratios of $v_{2} \mathrm{PO}_{4}{ }^{3-} /$ Amide III and $v_{4} \mathrm{PO}_{4}{ }^{3-} /$ Amide III (Fig. 2a and b) do not change significantly for the horizontal and vertical polarization of incident light. As a result, the maps of these ratios could be used to give the information on the relation of the mineral and organic components content in the spongy bone tissue. Similarly, no considerable differences were noted for the ratio of $v_{1} \mathrm{CO}_{3}{ }^{2-} / v_{2} \mathrm{PO}_{4}{ }^{3-}$ ratio (Fig. 2d) for the horizontal and vertical polarization of laser light. However, the $v_{1} \mathrm{CO}_{3}{ }^{2-} / v_{2} \mathrm{PO}_{4}{ }^{3-}$ ratio reveals more pronounced differences for the above two polarizations, but is not sensitive to fibers orientation. Thus the maps of $v_{1} \mathrm{CO}_{3}{ }^{2-} / v_{2} \mathrm{PO}_{4}{ }^{3-}$ ratio inform about the relative content of two mineral components, carbonates to phosphates, in the same area of the spongy bone tissue. The collagen orientation in trabecula is shown on the maps of $v_{1} \mathrm{PO}_{4}{ }^{3-} /$ Amide I ratio because these bands are quite sensitive to the orientation effect. The line plot of 

III (a), $v_{4} \mathrm{PO}_{4}{ }^{3-} /$ Amide III (b), $v_{1} \mathrm{PO}_{4}{ }^{3-} /$ Amide $\mathrm{I}(\mathbf{c})$ and $v_{1} \mathrm{CO}_{3}{ }^{2-} / v_{2} \mathrm{PO}_{4}{ }^{3-}$ (d) ratios obtained from Raman maps of spongy bone tissue with horizontal (thin gray line) and vertical (thick black line) polarization of laser light. The photo (e) with grid of Raman spectra measurements on trabecula surface. The lines show directions of successive measurements
Fig. 2 Plots of $v_{2} \mathrm{PO}_{4}{ }^{3-} /$ Amide
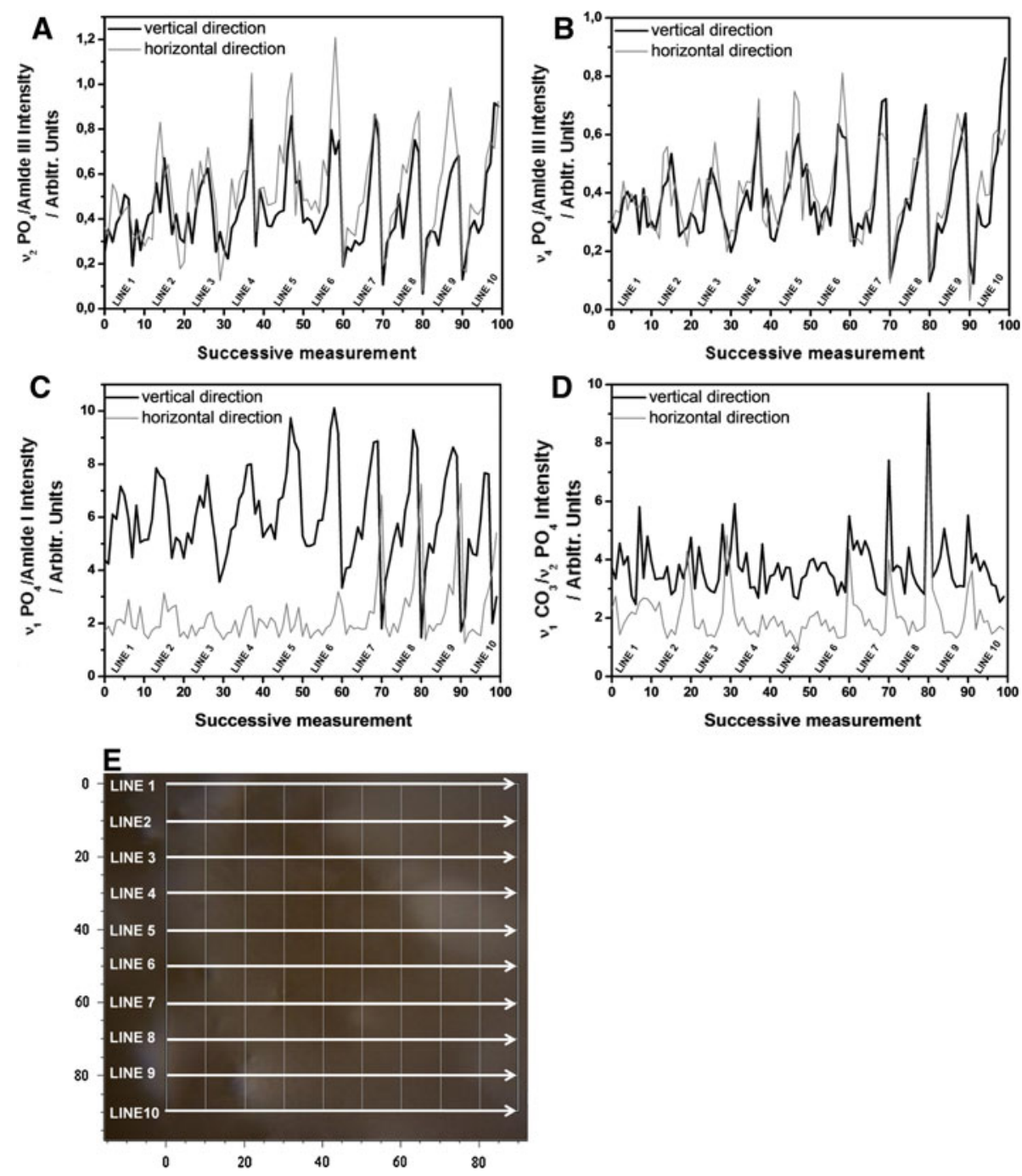

$v_{1} \mathrm{PO}_{4}{ }^{3-}$ /Amide I ratio (Fig. 2c) displays pronounced orientational effect, which is not observed in the other ratio plots. Changes in the fibers orientation in the trabecula surface studied appear in the seventh line. Then the $v_{1} \mathrm{PO}_{4}{ }^{3-} /$ Amide I ratio decreases for the vertical polarization of incident light and increases for the horizontal polarization. In the ratio plot, the orientation effect connected with collagen fibers arrangement is visible due to the phase differences between $v_{1} \mathrm{PO}_{4}{ }^{3-}$ and Amide I components appearing because their vibration directions are perpendicular to each other. The highest value of $v_{1} \mathrm{PO}_{4}{ }^{3-} /$ Amide I ratio is obtained when the polarization of incident light is parallel to the collagen fiber orientation, in contrast, the lowest value of $v_{1} \mathrm{PO}_{4}{ }^{3-}$ /Amide $\mathrm{I}$ ratio is obtained when the polarization of incident light is perpendicular to the collagen fiber orientation. Consequently, the maximum ratio of $v_{1} \mathrm{PO}_{4}{ }^{3-} /$ Amide $\mathrm{I}$ in the vertical polarization corresponds to the minimum value of this ratio in the horizontal polarization (Fig. 2c)
Raman spectroscopy with a micro-level spatial resolution allows generation of images mapping the Raman spectra and in consequence permits identification of local variations in composition and structure of bone tissue [32]. Figures 3 and 4 present images of the ratios of $v_{2} \mathrm{PO}_{4}{ }^{3-}$ / Amide III (a, e), $v_{4} \mathrm{PO}_{4}{ }^{3-} /$ Amide III $(\mathrm{b}, \mathrm{f}), v_{1} \mathrm{CO}_{3}{ }^{2-}$ / $v_{2} \mathrm{PO}_{4}{ }^{3-}(\mathrm{c}, \mathrm{g})$ and $v_{1} \mathrm{PO}_{4}{ }^{3-} /$ Amide $\mathrm{I}(\mathrm{d}, \mathrm{h})$. The maps of Raman spectra generated for the vertical and horizontal polarization of incident light are presented in Fig. 3 and those generated for different polarizations of the incident and scattered light are given in Fig. 4 (the arrows in figures indicate these polarization directions). Each image displays the same area of trabecula surface. In the scale bars, the maximum values of the ratios are specified and the minimum value is 0 for all images. The bright contrast corresponds to the maximum ratio, while dark to the lowest one.

Conclusions on the chemical composition can be drawn from Fig. 3 showing the ratios $v_{2} \mathrm{PO}_{4}{ }^{3-} /$ Amide III (a), $v_{4} \mathrm{PO}_{4}{ }^{3-}$ /Amide III (b) and $v_{1} \mathrm{CO}_{3}{ }^{2-} / v_{2} \mathrm{PO}_{4}{ }^{3-}$ (c) obtained 

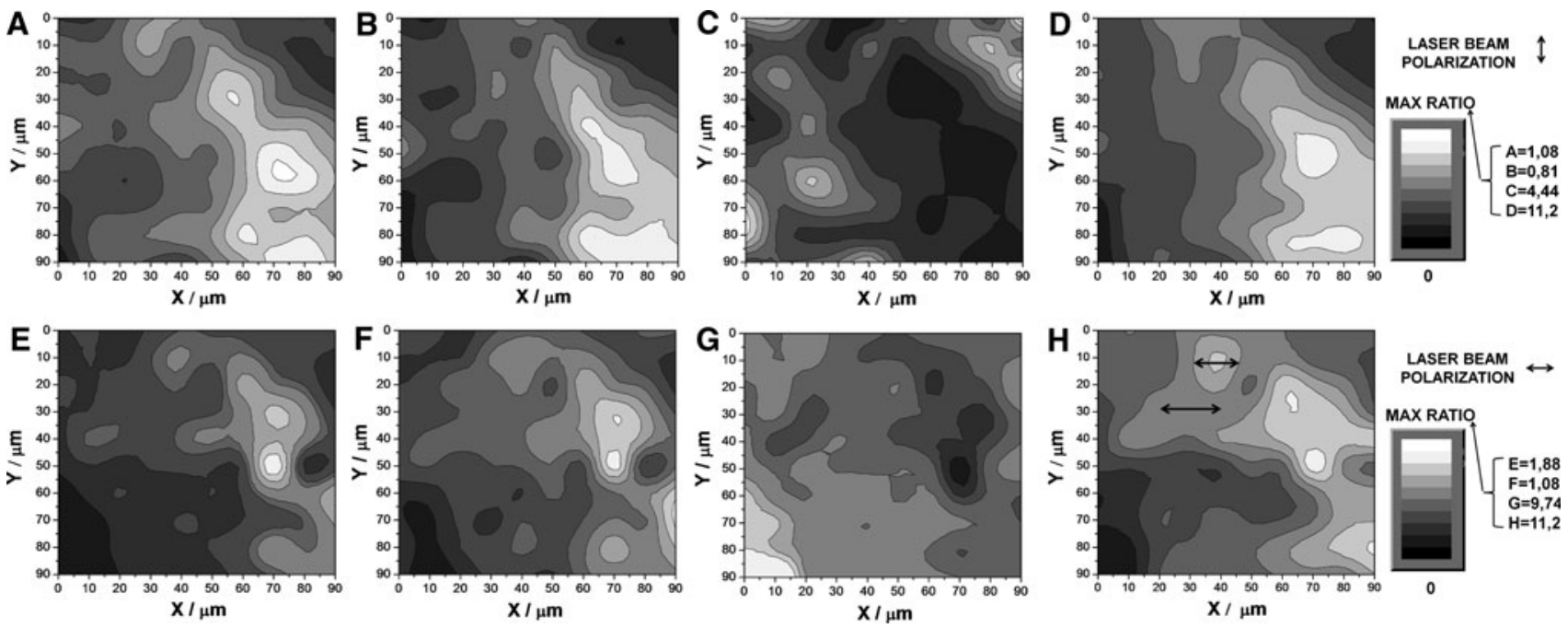

Fig. 3 The contrast images based on the ratios of $v_{2} \mathrm{PO}_{4}{ }^{3-} /$ Amide III (a, e), $v_{4} \mathrm{PO}_{4}{ }^{3-} /$ Amide III $(\mathbf{b}, \mathbf{f}), v_{1} \mathrm{CO}_{3}{ }^{2-} / v_{2} \mathrm{PO}_{4}{ }^{3-}(\mathbf{c}, \mathbf{g})$ and $v_{1} \mathrm{PO}_{4}{ }^{3-} /$ Amide I $(\mathbf{d}, \mathbf{h})$. Arrows indicate polarization of laser light, and the color bar displays the maximum ratio for each image
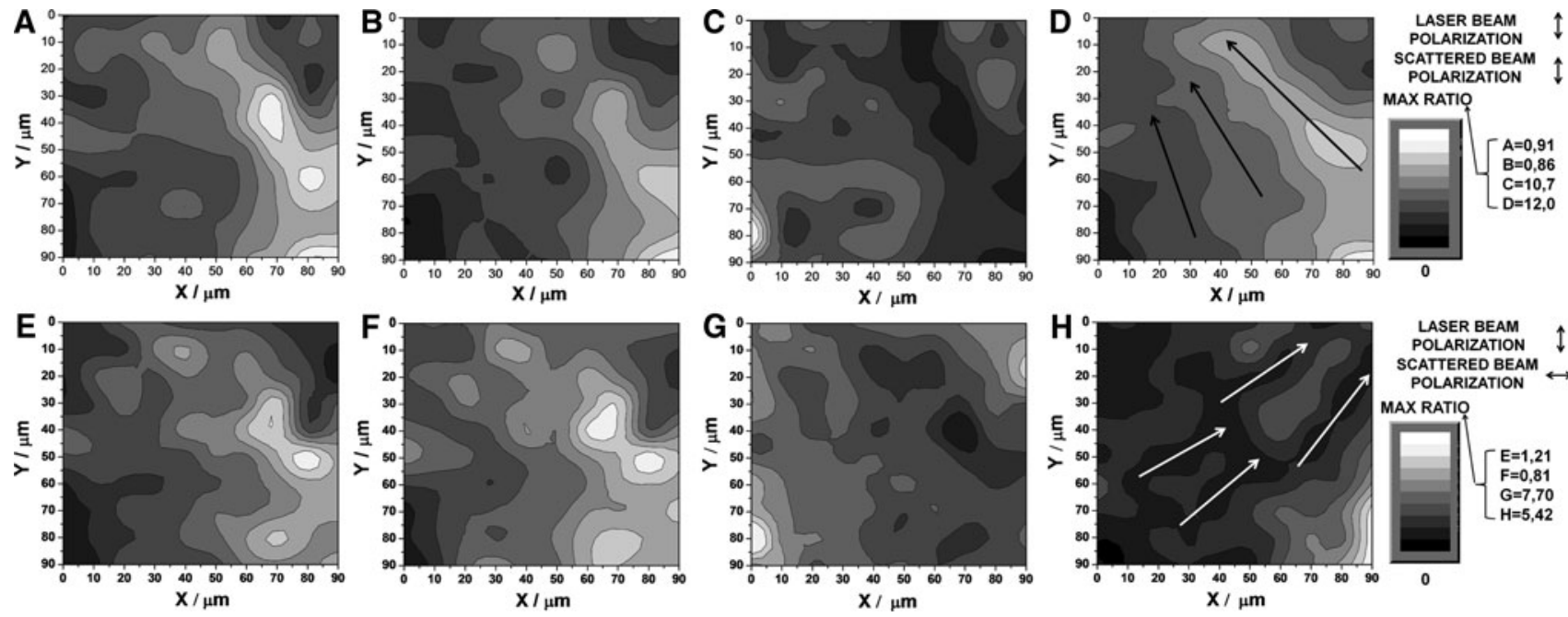

Fig. 4 The contrast images based on the ratios of $v_{2} \mathrm{PO}_{4}{ }^{3-} /$ Amide III (a, e), $v_{4} \mathrm{PO}_{4}{ }^{3-} /$ Amide III (b, f), $v_{1} \mathrm{CO}_{3}{ }^{2-} / v_{2} \mathrm{PO}_{4}{ }^{3-}(\mathbf{c}, \mathbf{g})$ and $v_{1} \mathrm{PO}_{4}{ }^{3-} /$ Amide I $(\mathbf{d}, \mathbf{h})$. Arrows indicate polarisation of laser incident and scattered beams, and the color bar displays the maximum ratio for each image

for the vertical polarization of laser light. The images in Fig. $3 a, b$ indicate the degree of mineralization and give information about changes in the content of hydroxyapatite crystals with respect to that of collagen in the trabecula. The images present very similar contrast changes; i.e., brighter and darker areas are in the same regions and correspond to higher and lower ratios of mineral to organic content. In Fig. 3c we compare the content of carbonate apatite to that of hydroxyapatite. The higher ratio of phosphate to carbonate crystals occurs in the same area as the higher ratio of hydroxyapatite to collagen content in Fig. 3a, b. Figure $3 \mathrm{~d}$ displays $v_{1} \mathrm{PO}_{4}{ }^{3-} /$ Amide I ratio contrast image of the bands quite sensitive to the orientation effect. This image should allow determination of the collagen fiber orientation in trabecula, but it does not show a pronounced structural effect. Figure $3 d$ reveals slight contrast changes in comparison with those in the other ratio images referring to the chemical composition (Fig. 3a-c). The similarity in the character of the maps referring to the collagen orientation and chemical composition is probably related to the distribution of bone tissue constituents. This means that differences in contrast in the maps referring to chemical composition are too large with respect to those referring to collagen fibers orientation so the orientation effect is undetectable. Hence, Fig. 3a-d present very similar contrast images.

Figure 3 displays images for $v_{2} \mathrm{PO}_{4}{ }^{3-} /$ Amide III (e), $v_{4} \mathrm{PO}_{4}{ }^{3-}-$ Amide III (f), $v_{1} \mathrm{CO}_{3}{ }^{2-} / v_{2} \mathrm{PO}_{4}{ }^{3-}$ (g) and $v_{1} \mathrm{PO}_{4}{ }^{3-} /$ Amide I (h) ratios obtained for the horizontal polarization of laser light. The ratio images illustrating chemical 
composition (Fig. 3e-g) are similar and the contrasts in them change a similar way. The small differences in contrast are justified because the bands used in these ratios are less sensitive to orientation effect. The images obtained in the orthogonal polarization-vertical and horizontal-are quite similar and do not show any significant differences. The slight changes in the images based on the ratios $v_{2} \mathrm{PO}_{4}{ }^{3-}$ /Amide III, $v_{4} \mathrm{PO}_{4}{ }^{3-} /$ Amide III, $v_{1} \mathrm{CO}_{3}{ }^{2-} / v_{2} \mathrm{PO}_{4}{ }^{3-}$ are a result of different behavior of the Amide III band in light of different polarizations [33]. However, the changes in the Amide III band are much smaller than in that of Amide I, so only the first band gives information about organic composition. Fig. $3 \mathrm{~h}$ based on the bands sensitive to collagen fiber orientation shows contrast changes similar to those in the maps referring to chemical composition in trabecula (Fig. 3e-g). Therefore, it is difficult to conclude if the highest contrast corresponds to the orientation of collagen fibers or to the changes in chemical composition. Moreover, the images in Fig. $3 \mathrm{~d}$ and $\mathrm{h}$ are almost identical and their contrasts do not change much for different light polarizations. Perhaps, such small changes in contrast result from a particular orientation of collagen fibers on this surface. If the orientation is not parallel to the horizontal or vertical polarization of laser light, so if the fibers are skewed, then the changes in polarizability of collagen molecule are detected for both light polarization. Therefore, the ratio images obtained for vertical and horizontal polarizations are very similar. Detailed comparative analysis of Fig. $3 d$ and $h$ reveals small differences in contrast. Arrows in Fig. 3h indicate the area with bright color contrast that corresponds to the dark contrast areas in Fig. 3d. It could mean that collagen orientation is parallel to horizontal direction in this part of trabecula surface. In the other sites of the images, no distinct differences in contrast appear, so it is impossible to say anything more about collagen orientation.

Images in Fig. 4 were obtained for appropriate different polarizations of the incident and scattered light. Fig. $4 a-d$ were obtained for the vertical polarization of the incident and scattered beams, while Fig. 4e-h for their orthogonal polarization. The maps based on the ratios of $v_{2} \mathrm{PO}_{4}{ }^{3-} /$ Amide III $(\mathrm{a}, \mathrm{e}), v_{4} \mathrm{PO}_{4}{ }^{3-} /$ Amide III $(\mathrm{b}, \mathrm{f})$ do not show considerable changes, so these images were taken as an indicator of mineral content to organic content in spongy bone tissue. The images based on the ratios of $v_{1} \mathrm{CO}_{3}{ }^{2-}$ / $v_{2} \mathrm{PO}_{4}{ }^{3-}$ (c, g) do not show distinct changes for different polarizations of scattered light, similarly as those of $v_{2} \mathrm{PO}_{4}{ }^{3-} /$ Amide III and $v_{4} \mathrm{PO}_{4}{ }^{3-} /$ Amide III, thus they also refer to the spongy bone composition, i.e., give the information about relative amount of carbonate apatite to hydroxyapatite. So small changes in Fig. $4 \mathrm{a}-\mathrm{c}$, e-g prove that $v_{2} \mathrm{PO}_{4}{ }^{3-}, v_{4} \mathrm{PO}_{4}{ }^{3-}, v_{1} \mathrm{CO}_{3}{ }^{2-}$ and Amide III bands are less susceptible to polarization effects. Unlike these bands, those of $v_{1} \mathrm{PO}_{4}{ }^{3-}$ and Amide I are characterized by higher sensitivity to polarization effects, therefore the ratio of $v_{1} \mathrm{PO}_{4}{ }^{3-}$ /Amide I could show the fiber orientation. Contrast images in Fig. 3d, h obtained for the incident light polarized in two different directions do not reveal considerable differences, whereas the contrast images in Fig. 4d, $\mathrm{h}$ obtained for the polarized incident and scattered laser beams display pronounced changes. When the polarization of laser incident and scattered light is the same, the contrast in (Fig. 4d) is similar to that in the images which give information about the bone composition (Fig. 4a, b). On the other hand, when the polarizations of incident and scattered light are mutually perpendicular, then the contrast (Fig. 4h) changes a lot. Such different behavior for the polarized scattered light is a result of the presence of collagen fibers in the structure of spongy bone tissue. A certain area on the trabecula surface corresponds to high intensity values (bright contrast) in Fig. $4 \mathrm{~d}$ and the same region in Fig. 4h corresponds to low intensity values (dark contrast). Changes in intensity ratio induced by changes in the polarization of scattered light allow determination of the orientation of collagen fibers. For example, we can conclude that the collagen fibers are arranged in parallel to the incident beam polarization (or scattered beam polarization) only when the polarizations of these two beams are the same, which corresponds to bright color contrast. However, to verify this conclusion, the area of bright contrast must correspond to the area of dark contrast on the map for the orthogonal polarizations of the incident and scattered light (Fig. 4h). The analysis of collagen orientation in trabecula is performed on the basis of Fig. $4 \mathrm{~d}, \mathrm{~h}$. The arrows in Fig. 4d indicate collagen fibers arrangement, which is close to vertical direction. The arrows in Fig. $4 \mathrm{~h}$ indicate the region of dark contrast, so taking into account the above, it is possible to conclude that the collagen fibers are arranged in perpendicular to these arrows. The results allow concluding that $v_{1} \mathrm{PO}_{4}{ }^{3-} /$ Amide $\mathrm{I}$ ratios permit determination of the orientations of collagen fibers in spongy bone tissue.

\section{Conclusion}

This work presents possibilities of Raman spectroscopy application for determination of chemical composition and orientation of collagen fibers in human spongy bone tissue. Raman microspectroscopy had been employed to study organization of collagen fibers and material composition in cortical bone by Kazanci et al. [25]. This study shows that the Raman spectral maps allow determination of local variations in mineral and organic content in the human spongy bone and changes in orientation of collagen fibers on the bone surface. Analysis of the ratios of 
$v_{2} \mathrm{PO}_{4}{ }^{3-}$ /Amide III, $v_{4} \mathrm{PO}_{4}{ }^{3-} /$ Amide III and $v_{1} \mathrm{CO}_{3}{ }^{2-}$ / $v_{2} \mathrm{PO}_{4}{ }^{3-}$ could be used to conclude about the relative amount of bone tissue components. In this study, it is not important which polarization of laser beam is used, because these ratios are less sensitive to the orientation effect. Changes in the ratio of $v_{1} \mathrm{PO}_{4}{ }^{3-} /$ Amide $\mathrm{I}$ for the polarized incident and scattered light allow determination of the arrangement of collagen fibers in trabecula. The ratio maps obtained for the same polarization of incident and scattered light give information about the orientation of collagen fibers, but also on the distribution of the material components. The ratio maps obtained in the orthogonal polarization of incident and scattered light reveal the sites at which the ratios change in comparison with the ratio maps obtained in the same polarization. This process separates the orientational and compositional changes and permits identification of the sites of collagen fibers occurrence in trabecula.

The versatility of the Raman method as the analytical spectroscopic technique offers a possibility to get insights into the organization of bone. Raman spectroscopy with micro-level spatial resolution permits detection of local variations in composition and structure of the spongy bone tissue. The above information in combination with evaluation of bone mineral density could allow earlier detection of fracture risk. Understanding the bone tissue organization at the microstructural level can help finding the origins of bone diseases such as osteoporosis or osteoarthrosis.

Open Access This article is distributed under the terms of the Creative Commons Attribution Noncommercial License which permits any noncommercial use, distribution, and reproduction in any medium, provided the original author(s) and source are credited.

\section{References}

1. Centrella M, McCarthy TL, Canalis E. Skeletal tissue and transforming growth factor beta. Fed Am Soc Exp Biol J. 1988;2: 3066-73.

2. Buckwalter JA, Glimcher MJ, Cooper RR, Recker R. Bone biology part I: structure, blood supply, cells, matrix, and mineralization. J Bone Joint Surg. 1995;77:1256-75.

3. Buckwalter JA, Glimcher MJ, Cooper RR, Recker R. Bone biology part II: formation, form, modeling, remodeling, and regulation of cell function. J Bone Joint Surg. 1995;77:1276-89.

4. Diaz-Flores L, Gutierrez R, Lopez-Alonso A, Gonzalez R, Varela $\mathrm{H}$. Pericytes as a supplementary source of osteoblasts in periosteal osteogenesis. Clin Orthop. 1992;275:280-6.

5. Harada S, Rodan GA. Control of osteoblast function and regulation of bone mass. Nature. 2003;423:349-55.

6. Lawton DM, Andrew JG, Marsh DR, Hoyland JA, Freemont AJ. Expression of the gene encoding the matrix gla protein by mature osteoblasts in human fracture non-unions. Mol Pathol. 1999;52: $92-6$.

7. Sierpowska J, Lammi MJ, Hakulinen MA, Jurvelin JS, Lappalainen R, Toyras J. Effect of human trabecular bone composition on its electrical properties. J Med Eng Phys. 2007;29:845-52.
8. Boivin G, Meunier PJ. Effects of bisphosphonates on matrix mineralization. J Musculoskelet Neuronal Interact. 2002;2:53843.

9. Favus MJ. Primer on the metabolic bone diseases and disorders of mineral metabolism. 5th ed. Washington, DC: American Society for Bone and Mineral Research; 2003.

10. De Carmejane O, Morris MD, Davis MK, Stixrude L, Tecklenburg M, Rajachar RM, Kohan DH. Bone chemical structure response to mechanical stress studied by high pressure Raman spectroscopy. Calcif Tissue Int. 2005;76:207-13.

11. Fratzl P, Gupta HS, Paschalis EP, Roschger P. Structure and mechanical quality of the collagen-mineral nano-composite in bone. J Mater Chem. 2004;14:2115-23.

12. Favus MJ. Primer on the metabolic bone diseases and disorders of mineral metabolism. 4th ed. Philadelphia: Lippincott Williams \& Wilkins; 1999.

13. Canalis E, McCarthy T, Centrella M. Growth factors and the regulation of bone remodelling. J Clin Invest. 1988;81:277-81.

14. Bonjour JP, Burckhardt P, Dambacher M, Kraenzlin ME, Wimpfheimer C. Epidemiology of osteoporosis. Schweiz Med Wochenschr. 1997;127:659-67.

15. Bonnaire F, Zenker H, Lill C, Weber AT, Linke B. Treatment strategies for proximal femur fractures in osteoporotic patients. Osteoporos Int. 2005;16:93-102.

16. Penel G, Delfosse C, Descamps M, Leroy G. Composition of bone and apatitic biomaterials as revealed by intravital Raman microspectroscopy. Bone. 2005;36:893-901.

17. Gremlich H-U, Yan B. Infrared and Raman spectroscopy of biological materials. New York: Marcel Decker; 2001.

18. Timlin JA, Carden A, Morris MD. Chemical microstructure of cortical bone probed by Raman transects. Appl Spectrosc. 1999;53: 1429-35.

19. Timlin JA, Carden A, Morris MD, Rajachar RM, Kohn DH. Raman spectroscopic imaging markers for fatigue-related microdamage in bovine bone. Anal Chem. 2000;72:2229-36.

20. Mantsch H, Jackson M. Molecular spectroscopy in biodiagnostics (from Hippocrates to Herschel and beyond). J Mol Struct. 1995; 347:187-206.

21. Akkus O, Adar F, Schaffler MB. Age-related changes in physicochemical properties of mineral crystals are related to impaired mechanical function of cortical bone. Bone. 2004;34:443-53.

22. Morris MD, Finney WF. Recent developments in Raman and infrared spectroscopy and imaging of bone tissue. Spectroscopy. 2004;18:155-9.

23. Carden A, Rajachar RM, Morris MD, Kohn DH. Ultrastructural changes accompanying the mechanical deformation of bone tissue: a Raman imaging study. Calcif Tissue Int. 2003;72:16675.

24. Kazanci M, Roschger P, Paschalis EP, Klaushofer K, Fratzl P. Bone osteonal tissues by Raman spectral mapping: orientationcomposition. J Struct Biol. 2006;156:489-96.

25. Kazanci M, Wagner HD, Manjubala NI, Gupta HS, Paschalis E, Roschger P, Fratzl P. Raman imaging of two orthogonal planes within cortical bone. Bone. 2007;41:456-61.

26. Raghavan M, Sahar ND, Wilson RH, Mycek MA, Pleshko N, Kohn DH, Morris MD. Quantitative polarized Raman spectroscopy in highly turbid bone tissue. J Biomed Opt. 2010;15:037001.

27. Goodyear SR, Gibson IR, Skakle JMS, Wells RPK, Aspden RM. A comparison of cortical and trabecular bone from C57 black 6 mice using Raman spectroscopy. Bone. 2009;44:899-907.

28. Leroy G, Penel G, Leroy N, Bres E. Human tooth enamel: a Raman polarized approach. Appl Spectrosc. 2002;56:1030-4.

29. Rousseau ME, Lefèvre T, Beaulieu L, Asakura T, Pézolet M. Study of protein conformation and orientation in silkworm and spider silk fibers using Raman microspectroscopy. Biomacromolecules. 2004;5:2247-57. 
30. Tsuboi M, Benevides JM, Bondre P, Thomas GJ Jr. Structural details of the thermophilic filamentous bacteriophage PH75 determined by polarized Raman microspectroscopy. Biochemistry. 2005;44:4861-9.

31. Tsuda H, Arends J. Orientational micro-Raman spectroscopy on hydroxyapatite single crystals and human enamel crystallites. J Dent Res. 1994;73:1703-10.
32. Carden A, Morris MD. Application of vibrational spectroscopy to the study of mineralized tissues (review). J Biomed Opt. 2000;5: 259-68.

33. Bonifacio A, Sergo V. Effects of sample orientation in Raman microspectroscopy of collagen fibers and their impact on the interpretation of the amide III band. Vib Spectrosc. 2010;53: 314-7. 